\title{
Augmented robust T-S fuzzy control based PMSG wind turbine improved with $H_{\infty}$ performance
}

\author{
Naoual Tidjani ${ }^{1}$, Abderrezak Guessoum ${ }^{2}$ \\ ${ }^{1}$ Djilali Bounaama Khemis Miliana University, Khemis Miliana, Algeria \\ ${ }^{2}$ LATSI, University of Blida, Blida, Algeria
}

\begin{tabular}{l} 
Article Info \\
\hline Article history: \\
Received Aug 27, 2020 \\
Revised Jan 12, 2021 \\
Accepted Jan 30, 2021 \\
\hline
\end{tabular}

Keywords:

$H_{\infty}$ performance

PMSG

Takagi-Sugeno fuzzy control

PDC approach

Wind turbine

\begin{abstract}
In this paper, an improved augmented Takagi-Sugeno fuzzy control design applied to the system of converting wind turbine energy was proposed. The wind generator used is based on a permanent magnet synchronous wind power generator (PMSG) under varying operation of the wind speed. The proposed T-S fuzzy control strategy aims to maximize wind energy in low wind speed. A part of our contribution lies in the limitation of the power output of the wind generator in high wind speed. Through the concept of the virtual desired variables, the design of the output tracking controller is achieved. In light of this concept, the developed T-S fuzzy control was designed via parallel-distributed compensation (PDC) approach with $H_{\infty}$ performance.

Sufficient conditions for the stability of the closed-loop system affected by external disturbances are proved from Lyapunov's direct method and the feedback gains of the controller strategy are determined by linear matrix inequalities (LMIs) tools. Another contribution is in showing the robustness of the Takagi-Sugeno based control strategy, with a focus on a set of system parameters with model uncertainties. The simulation results show the high performance of the proposed controller strategy for a 5MW (PMSG) obtained through simulation.
\end{abstract}

This is an open access article under the CC BY-SA license.

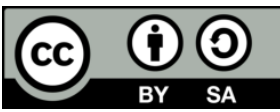

Corresponding Author:

Naoual Tidjani,

Djilali Bounaama Khemis Miliana University, Blida, Algeria

Email: n.tidjani@univ-dbkm.dz

\section{INTRODUCTION}

Nowadays, efforts by governments and businesses are further promoting to propel the mass adoption of renewable energy in order to steer the production of electricity from clean energy sources. Among these energies, the renewable wind energies are becoming used widely in this context, because it is cost-effective, sustainable, clean, and does not produce greenhouse gases.

Recently, many wind turbine technologies have been developed, where the main goal is to ensure a good quality of electrical power, and an optimization of the energy resource that is extracted from the wind. Therefore, the choice of the most appropriate electrical generator for a wind turbine is an important task. Appropriately, the most commonly used generators in wind power generation, are based on a permanent magnet synchronous generator (PMSG), for its many advantages compare to other machines [1]. The PMSG does not require electrical excitation, as the magnetic field is produced by permanent magnets, rather than by the coil. Hence, PMSG's does not need slip rings nor brushes, which reduces the weight, implementation costs, maintenance and no field copper losses. Furthermore, there is the possibility to avoid gearbox connection to the turbine. It will result in high dynamic performance, and high power with a wide operating 
speed range [2], [3]. As is well known, the complex dynamic behavior describing wind turbine systems, suggests robust control strategies to achieve the desired performance and ensure high stability [4], [5].

In previous findings, many control methods are proposed including linear controllers that are limited in the ability, to achieve acceptable control performances. Nonlinear controllers are better adapted to the wind energy conversion system (PMSG-WECS) under different wind speed variations [6]. Makhad et al. [7] design a novel integral backstepping strategy, to optimize wind energy in the event of abrupt changes in wind speed. Dahbi et al. [8] clarified the wind turbine system by combining maximum power point tracking (MPPT)-pitch angle control, using artificial neural networks to enable the network to be supplied with stable and efficient electrical energy. In the power system, the wind farm is more sensitive to disturbances. In this context, Chen et al. [9] designed MPPT control scheme based on nonlinear adaptive control to estimate the lumped perturbation term according to a high-gain perturbation observer. Moradi et al. [10] design a $H_{\infty}$ controller to improve the wind turbine power with robust stability and a less oscillatory behavior compared to the classical PID controller.

Moreover, fuzzy logic is well suited to modelling a nonlinear system according to [11]-[14]. In this context, Allouche et al. [15] designed a Takagi- Sugeno reference fuzzy model, in order to generate the optimal trajectory corresponding to only the maximum power. However, in that work, while also operating under time varying wind speed, the case of strong wind is not considered, despite the fact that the T-S fuzzy control can provide a wide large of control gain variation.

Based on the principles of the T-S fuzzy controller, which can provide an effective representation of complex and nonlinear systems, involving system uncertainties and external disturbances, the suggested solution in this work is to attenuate influence of external disturbances. In this article the tracking control design based on the augmented T-S fuzzy model by defining a lumped perturbation, is described using $H_{\infty}$ performance where the tracking error must be equal or less than a prescribed attenuation level. In this approach, the direct-drive permanent magnet wind turbine system is described by an aggregation of linear models, interconnected through membership functions. Then, local linear compensators for each subsystem to achieve the desired objective determine the global controller system.

Under a wide range of the wind speed, two different operations must be considered. The first one is a maximum power point tracking (MPPT) controller, established to optimize the generator speed. In this way, the captured energy is set at its maximum level, under rated wind speed. On the other hand, pitch angle operation control strategy is focused in limiting the turbine output power and the generator speed respectively, exceeding the wind speed rated value. Furthermore, in our approach, the model based T-S fuzzy controller is designed via parallel-distributed compensation (PDC), to determine the state feedback fuzzy controller, from the proposed augmented T-S fuzzy models. So, to improve the suggested controller, as a contribution, a stability criterion is derived from Lyapunov's direct method and, the $H_{\infty}$ tracking control performance can then be guaranteed at the same time. In this context, the feedback gains are solved very efficiently by convex optimization linear matrix inequalities (LMIs). Also, in order to provide optimal dynamic performance in term of convergence and robustness, the proposed method takes also the system parameter uncertainties of the mathematical model into account during the design process as another significant contribution.

In the following, section 2 includes a T-S fuzzy logic description for the wind turbine model based on the PMSG. In section 3, a tracking augmented T-S fuzzy controller is designed via the parallel distributed compensation (PDC) scheme and the Lyapunov stability criterion is presented for the closed-loop system to provide efficient power conversion. Then the robust tracking control performance $H_{\infty}$ is described, to guarantee the stability of the system and attenuate the disturbances, respectively. Linear matrix inequality (LMI) technique is adopted to solve for the feedback gains of the fuzzy controller. Section 4, presents simulation results applied for a $5 \mathrm{MW}$ permanent magnet synchronous generator. The conclusion and future scope close the article.

\section{WIND ENERGY CONVERSION SYSTEM}

\subsection{Wind energy model}

The mechanical power harnessed by a horizontal-axis wind turbine can be expressed in (1) [15]:

$$
P_{m}=\frac{1}{2} C_{p}(\lambda, \beta) \rho \pi R_{t}^{2} V_{w}^{3}
$$

where $R_{t}$ is the rotor radius, $\rho$ is the air density, $V_{w}$ is the wind speed and $C_{p}(\lambda, \beta)$ is a power coefficient presented as a nonlinear function that depends on tip speed ratio $\lambda$ and the pitch angle of the blades $\beta$ as shown in Figure 1. 
The empirical and nonlinear equation of $C_{p}(\lambda, \beta)$ based on the turbine characteristics are given by the expression in (2) [16]:

$$
\begin{aligned}
& C_{p}(\lambda, \beta)=0.73\left(\frac{151}{A}-0.58 \beta-0.02 \beta^{2.14} \rho-13.2\right) e^{-\frac{18.4}{A}} \\
& A=\frac{1}{\frac{1}{\lambda-0.02 \beta}-\frac{0.003}{\beta^{3}+1}}
\end{aligned}
$$

The tip speed ratio is defined as (3):

$$
\lambda=\frac{\Omega_{m} R_{t}}{V_{m}}
$$

where $\Omega_{\mathrm{m}}$ is the turbine speed.

The typical variations of $C_{p}(\lambda, \beta)$ from (2) with the tip-speed ratio $\lambda$ for various values of pitch angle $\beta$ are illustrated in Figure 1.

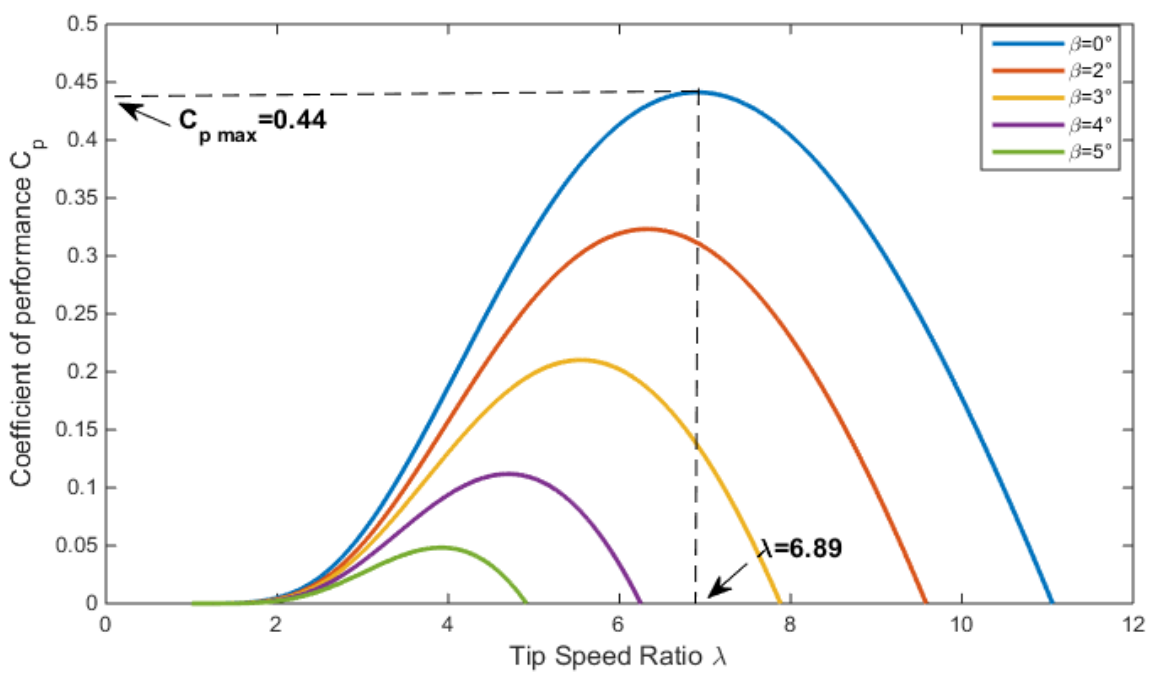

Figure 1. Coefficient of performance as a function of Tip Speed Ratio

The PMSG speed control based on MPPT (maximum power point tracking) control consists in maximizing the power converted. Thus, the optimal speed of the turbine must be adjusted as (4) [17], [18]:

$$
\Omega_{m o p t}=\frac{\lambda_{o p t} V_{w}}{R_{t}}
$$

for each wind speed with maximum $C_{p}=0.44$ and optimal $\lambda=6.89$ as shown in Figure 1 .

The purpose of the MPPT can therefore be provided by setting the electromagnetic power extracted from the wind at the maximum value given by (5) [15]:

$$
\mathrm{P}_{\max }=\frac{1}{2} \frac{\rho \pi R_{t}^{5} \mathrm{C}_{\text {max }}}{\lambda_{\text {opt }}^{3}} \Omega_{m}^{3}
$$

When the wind velocities are higher than rated, the blades are rotated by a control device based on pitch control as shown in Figure 2, to reduce the aerodynamic power captured by the wind and maintain the output power of PMSG at its rated value [19], [20]. 


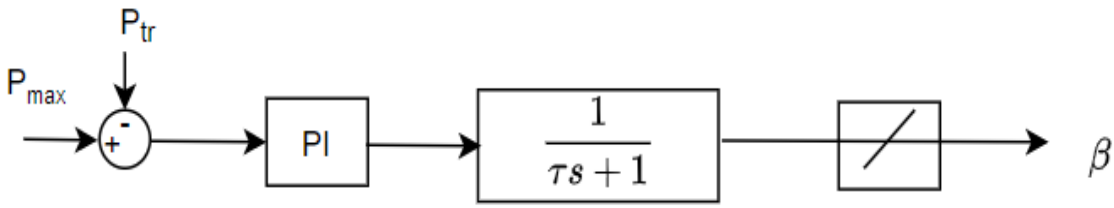

Figure 2. Pitch angle controller

\subsection{PMSG model}

For a non-salient PM machine, the dynamic model of the PMSG with rotational topology is given in the d-q synchronous frame using the Park's transformation as defined in (6) [16]:

$$
\left\{\begin{array}{c}
V_{d}=R i_{d}+L_{d} \frac{d i_{d}}{d t}-w_{e} L_{q} i_{q} \\
V_{q}=R i_{q}+L_{q} \frac{d i_{q}}{d t}+w_{e}\left(L_{d} i_{d}+\phi_{f}\right)
\end{array}\right.
$$

Where $V_{d}, V_{q}$ are the stator voltages in the d-q axis, $i_{d}, i_{q}$ are the currents in the d-q axis, $w_{e}=p \Omega_{m}$ is the electrical rotation speed, $R$ is the stator resistance, $\Phi_{f}$ the flux linkage of permanent magnets and $\mathrm{p}$ is the number of pole pairs. The dynamic equation of the wind turbine is given by (7) [16]:

$$
J \frac{d \Omega_{m}}{d t}=T_{e}-T_{m}-f \Omega_{m}
$$

$\mathrm{J}$ is equal to the summation of inertia moments of the turbine and the generator, $f$ is the friction coefficient, $T_{e}$ and $T_{m}$ prensents respetively the electromagnetic and aerodynamic torque.

The equation of the electromagnetic torque produced by the machine is defined as (8):

$$
T_{e}=\frac{3}{2} p \phi_{f} i_{q}
$$

Using (6) and (7), the dynamic model of the PMSG-WT in d-q reference frame can be described in the following nonlinear state space form (9) [24]:

$$
\left\{\begin{array}{c}
\dot{x}(t)=f(x(t), u(t))+g(x(t)) w(t) \\
y(t)=q(x(t))
\end{array}\right.
$$

where $x \in R^{n}$ is the state vector, $u \in R^{m}$ is the control input vector, $w(t)$ denotes the external disturbance, $y \in R^{m}$ is the mesured output, $f(x), g(x)$ and $q(x)$ are nonlinear with appropriate dimensions.

In order to express the nonlinear model of the machine as a T-S fuzzy model, with the measurable parameters as decision variables, (6) is described in the following nonlinear state space form (10) [24], [25]:

$$
\left\{\begin{array}{c}
\dot{x}(t)=A\left(\Omega_{m}\right) x(t)+B u(t)+E w(t) \\
y(t)=C x(t)
\end{array}\right.
$$

where

$$
\begin{aligned}
& x(t)=\left[i_{d}(t) i_{q}(t) \Omega_{m}(t)\right]^{T} \\
& A\left(\Omega_{m}\right)=\left[\begin{array}{ccc}
-\frac{R}{L_{d}} & p \Omega_{m} & 0 \\
-p \Omega_{m} & -\frac{R}{L_{q}} & -\frac{p \Phi_{f}}{L_{q}} \\
0 & \frac{3}{2} \frac{p \Phi_{f}}{J} & -\frac{f}{J}
\end{array}\right], B=\left[\begin{array}{cc}
\frac{1}{L_{d}} & 0 \\
0 & \frac{1}{L_{q}} \\
0 & 0
\end{array}\right], E=\left[\begin{array}{c}
0 \\
0 \\
-\frac{1}{J}
\end{array}\right], C=\left[\begin{array}{lll}
0 & 0 & 1
\end{array}\right], u(t)=\left[\begin{array}{l}
V_{d}(t) \\
V_{q}(t)
\end{array}\right]
\end{aligned}
$$




\subsection{Takagi-Sugeno (T-S) fuzzy model}

The T-S fuzzy model of PMSG is established based on the dynamic model given by (9). Thus, for ensuring the control of the stator converter side generator, the system is approximated by the T-S fuzzy dynamic model composed of $r$ rules in (11).

The $\mathrm{i}^{\text {th }}$ rule of the fuzzy model is as (13) [22], [23]:

$$
\text { IF } v_{1}(t) \text { is } M_{1 i} \text { and ... and } v_{j}(t) \text { is } M_{j i} T H E N \dot{x}(t)=A_{i} x(t)+B_{i} u(t)+E w(t)
$$

$i=1,2, \ldots, r ; M_{i j}$ denotes the fuzzy sets and represent the grade of membership of $v_{1}(t)$ in $M_{1 i}$. $r$ is the number of $I F \ldots$ THEN rule and $v_{i}(t)=\left(v_{1}, v_{2}, \ldots, v_{j}\right) \in \mathbb{R}^{j}$ are the premise variables [24] $A_{i} B_{i}$ are the local subsystem matrices.

Using the singleton fuzzifier, the resulting overall fuzzy system is inferred as (14) and (15)

$$
\begin{gathered}
\left\{\begin{array}{c}
\dot{x}(t)=\sum_{i=1}^{r} \mu_{i}(v(t))\left(A_{i} x(t)+B_{i} u(t)+E_{i} w(t)\right) \\
y(t)=\sum_{i=1}^{r} \mu_{i}(v(t)) C x(t)
\end{array}\right. \\
\mu_{i}(v(t))=\frac{M_{1 i}(v(t))}{\sum_{j=1}^{r} M_{1 j}(v(t))}
\end{gathered}
$$

Furthermore, it should be noted that the expression $\mu_{i}(v(t))$ for all i satisfy (16):

$$
\sum_{i=1}^{r} \mu_{i}(v(t)) \geq 0
$$

With $\sum_{i=1}^{r} \mu_{i}(v(t))=1$ for all $t>0$ where $\mu_{i}(v(t)) \geq 0$ for $i=1,2, \ldots, r$

Considering the rotor speed chosen as premise variable, two rules are deduced and the sub-matrices can be written as (17):

$$
\begin{aligned}
& A_{1}=\left[\begin{array}{ccc}
-\frac{R}{L_{d}} & p \Omega_{\max } & 0 \\
-p \Omega_{\max } & -\frac{R}{L_{q}} & -\frac{p \Phi_{f}}{L_{q}} \\
0 & \frac{3}{2} \frac{p \Phi_{f}}{J} & -\frac{f}{J}
\end{array}\right], A_{2}=\left[\begin{array}{ccc}
-\frac{R}{L_{d}} & p \Omega_{\min } & 0 \\
-p \Omega_{\min } & -\frac{R}{L_{q}} & -\frac{p \Phi_{f}}{L_{q}} \\
0 & \frac{3}{2} \frac{p \Phi_{f}}{J} & -\frac{f}{J}
\end{array}\right], \\
& B_{1}=B_{2}=\left[\begin{array}{cc}
\frac{1}{L_{d}} & 0 \\
0 & \frac{1}{L_{q}} \\
0 & 0
\end{array}\right], E_{1}=E_{2}=\left[\begin{array}{c}
0 \\
0 \\
-\frac{1}{J}
\end{array}\right]
\end{aligned}
$$

Figure 3 illustrates clearly the principle of the proposed fuzzy controller which corresponds to the maximum energy captured from the wind and its limitation at the nominal value.

In this regard, to simplify the tracking controller design, a set of new optimal trajectory is (18):

$$
\begin{aligned}
& x_{\text {opt }}(t)=\left[i_{\text {dopt }}(t) i_{\text {qopt }}(t) \Omega_{\text {mopt }}(t)\right]^{T} \\
& x(t)-x_{\text {opt }}(t) \rightarrow 0 \text { as } t \rightarrow 0 \\
& \tilde{x}(t)=x(t)-x_{\text {opt }}(t)
\end{aligned}
$$

$\tilde{x}(t)$ is defined as the tracking error and its time derivative is given by (19):

$$
\dot{\tilde{x}}(t)=\dot{x}(t)-\dot{x}_{o p t}(t)=\sum_{i=1}^{r} \mu_{i}(v(t))\left(A_{i} \tilde{x}(t)+B_{i} u(t)+E_{i} w(t)\right)-\dot{x}_{o p t}(t)
$$

The new fuzzy controller $\tau(\mathrm{t})$ is substituted in (14) is defined by $(20)$ :

$$
\sum_{i=1}^{r} \mu_{i}(v(t)) B_{i} \tau(t)=\sum_{i=1}^{r} \mu_{i}(v(t))\left(A_{i} x_{o p t}(t)+B_{i} u(t)\right)-\dot{x}_{\text {opt }}(t)
$$

(19) is rewritten in the following compact form (21):

$$
\dot{\tilde{x}}(t)=\sum_{i=1}^{r} \mu_{i}(v(t))\left(A_{i} \tilde{x}(t)+B_{i} \tau(t)+E_{i} w(t)\right)
$$




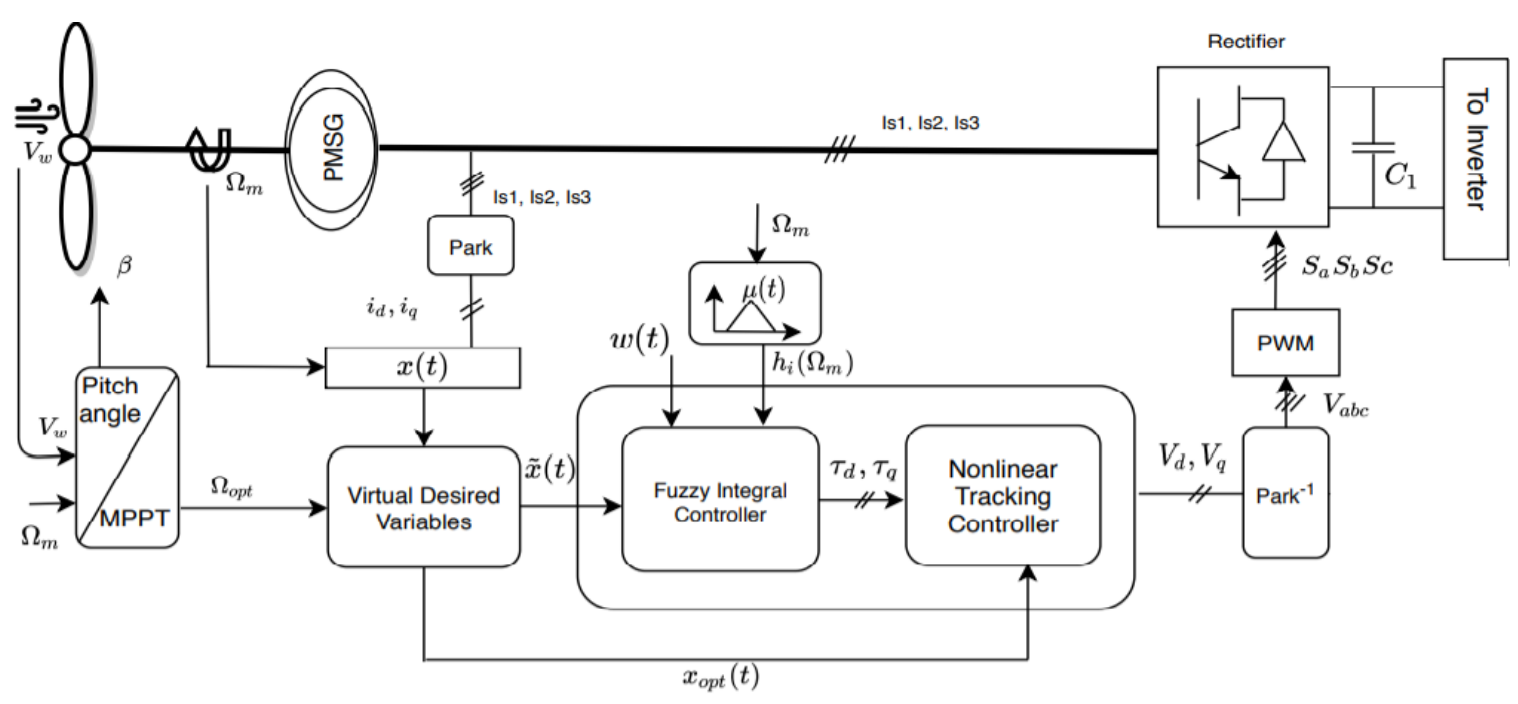

Figure 3. Generator-side control scheme

\section{ROBUST AUGMENTED T-S FUZZY CONTROLLER DESIGN}

In the next section, this controller is designed following the Parallel distributed compensation (PDC) technique employed for the T-S fuzzy model (21) [25], [27].

Hence, the $\mathrm{i}^{\text {th }}$ rule fuzzy controller can be described as (22):

$R^{i}: I F v_{1}(t)$ is $M_{1 i}$ and $\ldots$ and $v_{j}(t)$ is $M_{j i} \operatorname{THEN} \tau(t)=-K_{i} \tilde{x}(t)$, for $i=1,2, \ldots, r$

Where $K_{i} \in \mathbb{R}^{n * m}$ is the local state feedback vector. Then, the overall fuzzy PDC controller (22) is inferred as (23) [26]:

$\tau(t)=-\sum_{i=1}^{r} \mu_{i}(v(t)) K_{i} \tilde{x}(t)$

Substituting (23) into (21) yields the closed-loop fuzzy system as (24):

$\dot{\tilde{x}}(t)=\sum_{i=1}^{r} \sum_{j=1}^{r} \mu_{i}(v(t)) \mu_{j}(v(t))\left(A_{i}-B_{i} K_{j}\right) \tilde{x}(t)+E_{i} w(t)$

let us denote the integral state error vector as (25):

$\theta(t)=\int_{0}^{t} \tilde{x}(t) d t$

The augmented system containing the new fuzzy controller is presented as (26):

$\dot{\tilde{\tilde{X}}}(t)=\sum_{i=1}^{r} \mu_{i}(v(t))\left(\bar{A}_{i} \overline{\tilde{X}}(\mathrm{t})+\bar{B}_{i} \bar{\tau}(t)+\bar{E}_{i} \bar{W}(t)\right)$

Where the new feedback controller is inferred as (27):

$$
\bar{\tau}(t)=-\sum_{i=1}^{r} \mu_{i}(v(t)) K_{i} \tilde{x}(t)-\sum_{i=1}^{r} \mu_{i}(v(t)) F_{i} \theta(t)
$$

Thus, (27) is rewritten as (28):

$$
\bar{\tau}(t)=-\sum_{i=1}^{r} \mu_{i}(v(t))\left[K_{i} F_{i}\right]\left[\begin{array}{l}
\tilde{x}(t) \\
\theta(t)
\end{array}\right]
$$




$$
\bar{A}_{i}=\left[\begin{array}{cc}
A_{i} & 0 \\
I & 0
\end{array}\right], \bar{B}_{i}=\left[\begin{array}{c}
B_{i} \\
0
\end{array}\right], \bar{E}_{i}=\left[\begin{array}{c}
E_{i} \\
0
\end{array}\right], \bar{W}(t)=[w(t) 0]^{T}
$$

Substituting (28) into (26) yields the augmented closed-loop continuous T-S fuzzy model as (29):

$$
\dot{\tilde{\tilde{X}}}(t)=\sum_{i=1}^{r} \sum_{j=1}^{r} \mu_{i}(v(t)) \mu_{\mathrm{j}}(v(t))\left(\overline{\mathrm{G}}_{i j} \overline{\tilde{X}}(t)+\bar{E}_{i} \bar{W}(t)\right)
$$

\section{1. $H_{\infty}$ tracking control performance}

The optimality criterion by $H_{\infty}$ control performance consists to synthesize a controller such that the equilibrium of the closed-loop system (29) is stable and the attenuation of external disturbances is guaranteed considering the $H_{\infty}$ performance as (30) [25], [27]:

$$
\int_{0}^{t_{f}} \overline{\tilde{X}}^{T} d t \overline{\tilde{X}} \leq \overline{\tilde{X}}^{T}(0) \overline{\tilde{X}}(0)+\delta^{2} \int_{0}^{t_{f}} \bar{W}^{T}(t) \bar{W}(t) d t
$$

Where $t_{f}$ denotes the final times and $\delta$ is a specified disturbance constant.

In order to ensure the asymptotic stability of the augmented closed-loop model (29) guaranteeing the $H_{\infty}$ tracking performance described (30) for all disturbance, the gains $\bar{K}_{1}$ is obtained from $\overline{\mathrm{G}_{1 \mathrm{j}}}$.

\section{Lemma.}

The augmented closed-loop system described by (29) is asymptotically stable with the prescribed $H_{\infty}$ performance inequality (30), if and only if there exists a matrix $P=P^{T}>0$

such that (31)

$$
\left[\begin{array}{ccc}
\bar{A}_{l}{ }^{T} X+X \bar{A}_{l}{ }^{T}-\bar{B}_{l} M_{i}-M_{i}{ }^{T} B_{i}{ }^{T} & \bar{E}_{i} & X \\
\bar{E}_{i}{ }^{T} & -\delta^{2} I & 0 \\
X & 0 & -I
\end{array}\right]<0
$$

Proof.

Let the Lyapunov function for the augmented closed-loop system be defined as (32)

$$
V=\overline{\tilde{X}^{T}}(t) P \overline{\tilde{X}}(t)>0
$$

Taking the derivative $\dot{V}(\overline{\widetilde{X}}(t))$ will be required to satisfy (33) [23], [26]

$$
\dot{\tilde{X}^{T}}(t) P \overline{\tilde{X}}(t)+\overline{\tilde{X}^{T}} P \dot{\tilde{X}^{T}}(t)<0
$$

$H_{\infty}$ output tracking index $\delta$ is achieved for the resulting augmented closed-loop fuzzy if system (23) holds

$$
\dot{V}(\overline{\tilde{X}}(t)) \leq-\dot{\tilde{X}^{T}}(t) \overline{\tilde{X}}(t)+\delta^{2} \bar{W}(t)^{T} \bar{W}(t)<0
$$

Once the following matrix inequalities (for all i) are satisfied, i.e.

$$
\begin{aligned}
& \sum_{j=1}^{r} \mu_{i}(v(t))\left(\overline{\mathrm{G}}_{i j} \overline{\tilde{X}}(t)+\bar{E}_{i} \bar{W}(t)\right) P \overline{\tilde{X}}(t)+\overline{\tilde{X}}(t) P\left(\overline{\mathrm{G}}_{i j} \overline{\tilde{X}}(t)+\bar{E}_{i} \bar{W}(t)\right)+\overline{\tilde{X}^{T}}(t) \overline{\tilde{X}}(t)- \\
& \delta^{2} \bar{W}(t)^{T} \bar{W}(t)<0 \\
& \sum_{j=1}^{r} \mu_{i}(v(t))\left(\overline{\tilde{X}}(t)\left[\overline{G_{l}} P+\mathrm{P} \bar{G}_{l}\right]^{T} \overline{\tilde{X}}(t)+\bar{W}(t)\left[\bar{E}_{i} P\right] \overline{\tilde{X}}(t)+P \bar{E}_{i} \bar{W}(t)\right)-\delta^{2} \bar{W}(t)^{T} \bar{W}(t)<0 \\
& {\left[\begin{array}{cc}
\tilde{\tilde{X}}(t) \bar{W}(t)]\left[\begin{array}{cc}
\sum_{j=1}^{r} \mu_{i}(v(t)) \overline{G_{l}} P+\mathrm{P} \bar{G}_{l}^{T}+I & P \sum_{j=1}^{r} \mu_{i}(v(t)) \bar{E}_{i} \\
\sum_{j=1}^{r} \mu_{i}(v(t)) \bar{E}_{i}^{T} & -\delta^{2} I
\end{array}\right]\left[\begin{array}{l}
\overline{\tilde{X}}(t) \\
\bar{W}(t)
\end{array}\right]<0 \\
{\left[\begin{array}{cc}
\sum_{j=1}^{r} \mu_{i}(v(t))\left(\bar{G}_{l} P+\mathrm{P} \bar{G}_{l}^{T}\right)+I & P \sum_{j=1}^{r} \mu_{i}(v(t)) \bar{E}_{i} \\
\sum_{j=1}^{r} \mu_{i}(v(t)) \bar{E}_{i}^{T} P & -\delta^{2} I
\end{array}\right]<0}
\end{array}\right.}
\end{aligned}
$$


Hence, it suffices to check that

$$
\begin{aligned}
& {\left[\begin{array}{cc}
\left.\sum_{j=1}^{r} \mu_{i}(v(t)) \overline{\left(G_{l}\right.} P+\mathrm{P} \bar{G}_{\imath}^{T}\right) & P \sum_{j=1}^{r} \mu_{i}(v(t)) \bar{E}_{i} \\
\sum_{j=1}^{r} \mu_{i}(v(t)) \bar{E}_{i}^{T} P & -\delta^{2} I
\end{array}\right]+\left[\begin{array}{ll}
I & 0 \\
0 & 0
\end{array}\right]<0} \\
& {\left[\begin{array}{cc}
\left.\sum_{j=1}^{r} \mu_{i}(v(t)) \overline{\left(G_{l}\right.} P+\mathrm{P} \bar{G}_{\imath}^{T}\right) & P \sum_{j=1}^{r} \mu_{i}(v(t)) \bar{E}_{i} \\
\sum_{j=1}^{r} \mu_{i}(v(t)) \bar{E}_{i}^{T} P & -\delta^{2} I
\end{array}\right]+\left[\begin{array}{l}
I \\
0
\end{array}\right]\left[\begin{array}{ll}
I & 0
\end{array}\right]<0} \\
& {\left[\begin{array}{ccc}
\sum_{j=1}^{r} \mu_{i}(v(t))\left(\bar{G}_{l} P+\mathrm{P} \bar{G}_{l}^{T}\right) & P \sum_{j=1}^{r} \mu_{i}(v(t)) \bar{E}_{i} & I \\
\sum_{j=1}^{r} \mu_{i}(v(t)) \bar{E}_{i}{ }^{T} P & -\delta^{2} I & 0 \\
I & 0 & -I
\end{array}\right]<0} \\
& {\left[\begin{array}{ccc}
\bar{G}_{l} P+\mathrm{P} \bar{G}_{l}^{T} & P \bar{E}_{i} & I \\
\bar{E}_{i}^{T} P & -\delta^{2} I & 0 \\
I & 0 & -I
\end{array}\right]<0} \\
& {\left[\begin{array}{ccc}
P^{-1} & 0 & 0 \\
0 & I & 0 \\
I & 0 & 0
\end{array}\right]\left[\begin{array}{ccc}
\bar{G}_{l} P+\mathrm{P} \bar{G}_{\imath}{ }^{T} & P \bar{E}_{i} & I \\
\bar{E}_{i}{ }^{T} P & -\delta^{2} I & 0 \\
I & 0 & -I
\end{array}\right]\left[\begin{array}{ccc}
P^{-1} & 0 & 0 \\
0 & I & 0 \\
I & 0 & 0
\end{array}\right]<0}
\end{aligned}
$$

Thus, (43) is equivalent to (44)

$$
\left[\begin{array}{ccc}
\left.P^{-1} \overline{\left(G_{l}\right.} P+\mathrm{P} \bar{G}_{l}^{T}\right) P^{-1} & P^{-1} P \bar{E}_{i} & P^{-1} \\
\bar{E}_{i}{ }^{T} P P^{-1} & -\delta^{2} I & 0 \\
P^{-1} & 0 & -I
\end{array}\right]
$$

Let's consider $X=P^{-1}$ and $M_{i}=\bar{K}_{l} P^{-1}$; the control gains are obtaining by solving the LMI obtained by (45)

$$
\left[\begin{array}{ccc}
X \bar{A}_{l}^{T}+\overline{\mathrm{A}_{l}} \mathrm{X}-\bar{B}_{\imath} M_{i}-M_{i}{ }^{T} \bar{B}_{l}{ }^{T} & \bar{E}_{i} & X \\
\bar{E}_{i}{ }^{T} & -\delta^{2} I & 0 \\
X & 0 & -I
\end{array}\right]
$$

\subsection{Virtual disired variables system}

By using the fact that $\mathrm{g}(\mathrm{t})=\sum_{j=1}^{r} \mu_{i}(v(t))$ (20) is rewritten in the compact form (46) [25]:

$$
\begin{aligned}
g(t) B_{i} \tau(t)= & g(t)\left(A_{i} x_{o p t}(t)+B_{i} u(t)\right)-x_{o p t}(t) B(x(t))(\tau(t)-u(t))=A(x(t)) x_{o p t}(t)+ \\
& x_{\text {opt }}(t)
\end{aligned}
$$

where

$$
A_{i}(x(t))=g(t) A_{i}, B_{i}(x(t))=g(t) B_{i}
$$




$$
\left[\begin{array}{cc}
\frac{1}{L_{d}} & 0 \\
0 & \frac{1}{L_{q}} \\
0 & 0
\end{array}\right](u(t)-\tau(t))=-\left[\begin{array}{ccc}
-\frac{R}{L_{d}} & p \Omega_{m} & 0 \\
-p \Omega_{m} & -\frac{R}{L_{q}} & -\frac{p \Phi_{f}}{L_{q}} \\
0 & \frac{3}{2} \frac{p \Phi_{f}}{J} & -\frac{f}{J}
\end{array}\right]\left[\begin{array}{c}
i_{\text {dopt }}(t) \\
i_{\text {qopt }}(t) \\
\Omega_{\text {mopt }}(t)
\end{array}\right]+\left[\begin{array}{c}
l_{\text {dopt }}(t) \\
l_{\text {qopt }}(t) \\
\Omega_{\text {mopt }}(t)
\end{array}\right]
$$

In order to provide the maximum torque per ampere $i_{\text {dopt }}$ is always kept at zero. And from the measured optimal speed, $x_{o p t}(t)$ can be deduced from (48).

Therefore, the desired state variables for the tracking control are set to be

$$
x_{\text {opt }}(t)=\left[\begin{array}{c}
0 \\
\frac{2 J}{p \Phi_{f}}\left(\dot{\Omega}_{\text {mopt }}(t)+\frac{f}{J} \Omega_{\text {mopt }}(t)\right) \\
\Omega_{\text {mopt }}(t)
\end{array}\right]
$$

The required components of the rectified voltage vector are derived from the integral controllers for each d-q current component. Consequently, from (6) the machine voltages are described as (50):

$$
\left\{\begin{array}{c}
V_{d}=-p L_{q} \Omega_{m} i q_{o p t}+R i_{d o p t}+\imath \dot{d} \\
V_{q}=p \Phi_{f} \Omega_{m o p t}+R i_{q o p t}+L_{d} p \Omega_{m} i_{\text {dopt }}+\tau_{q}
\end{array}\right.
$$

\section{RESULTS AND DISCUSSION}

Matlab simpower system environment is employed to validate the elaborated model and control of the conversion system wind energy. The PMSG and turbine parameters are listed in Table 1.

Table 1. Specification of PMSG and turbine [14]

\begin{tabular}{cccc}
\hline PMSG parameters & Values & Wind turbine parameters & Values \\
\hline Generator rated power & $5 \mathrm{MW}$ & Turbine blade radius & $58 \mathrm{~m}$ \\
Generator rated voltage & $3.3 \mathrm{kV}$ & Optimal tip speed & 6.89 \\
Generator rated current & $1.515 \mathrm{kA}$ & Maximum $C_{p}$ value & 0.44 \\
Pole par number & 75 & The air density & $1.225 \mathrm{~kg} / \mathrm{m}^{2}$ \\
Generator stator resistance & $6.23 \mathrm{e}-003 \Omega$ & Rated wind speed & $12.12 \mathrm{~m} / \mathrm{s}$ \\
Generator $d-q$ axis inductance & $4.229 \mathrm{e}-003 \mathrm{H}$ & & \\
Permanent magnet flux & $11.1464 \mathrm{~Wb}$ & & \\
Generator inertia & $2 \mathrm{e}+5 \mathrm{Kg} . \mathrm{m}^{2}$ & & \\
\hline
\end{tabular}

The evolution of wind speed is illustrated in Figure 4, which shows that the speed varies around $10 \mathrm{~m} / \mathrm{s} \pm 30 \%$ during the time interval 0 to $60 \mathrm{~s}$.

Depending on the wind velocity variation, two operations can be triggered according to the appropriate control strategy.

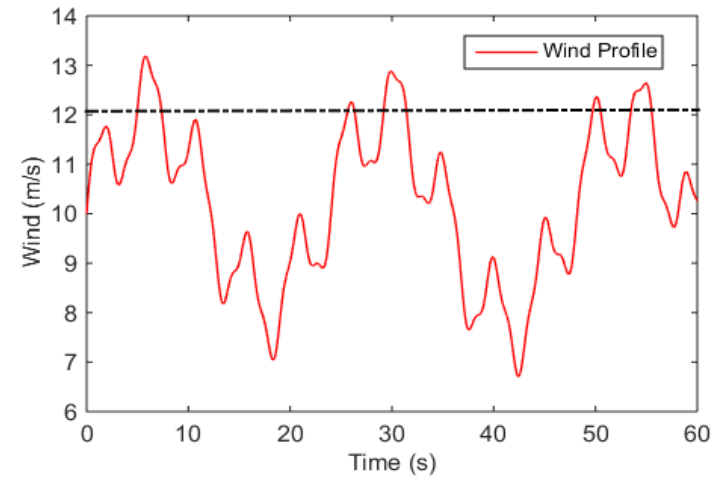

Figure 4. Wind Profile

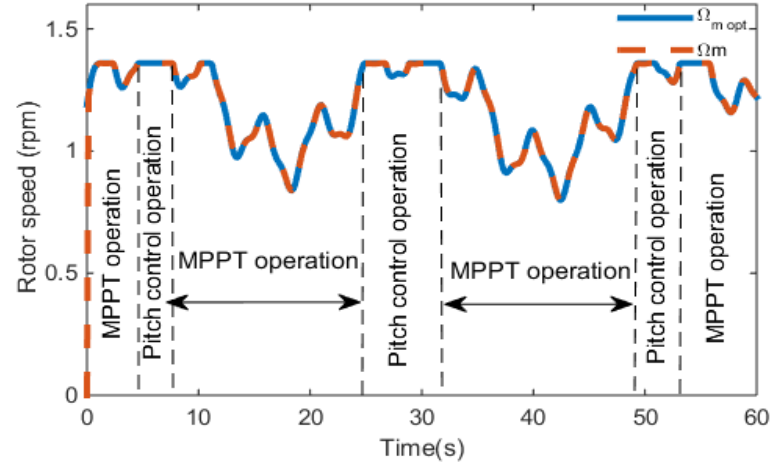

Figure 5. Generator rotor speed 
Figure 5 shows that below the rated wind speed, the generator speed is adjusted to be optimal when regulating the system under the specification of maximum power, and is limited at the rated value beyond the rated wind speed without overshooting. This shows the robustness of the method against the wind speed disturbance.

Figures 6(a) and 6(b) show that below the rated wind speed, the energy captured from the wind is maximized from MPPT operation, by adjusting the optimal rotor speed that corresponds to the maximum power. In this context, the power coefficient is kept constant at its maximum value at about 0.44 , and the pitch angle $\beta$ is preserved at zero for each MPPT operation, despite the variation of the wind.

Whereas beyond the rated wind speed value $V_{r}=12.12 \mathrm{~m} / \mathrm{s}$, the turbine is protected against damage and the blades are rotated by adjusting the pitch angle. As a result, $\beta$ increases with subsequent degradation of tip speed ratio $\lambda$ in order to reduce power coefficient $C_{p}$ to a value less than 0.44 , as shown at pitch control operation.

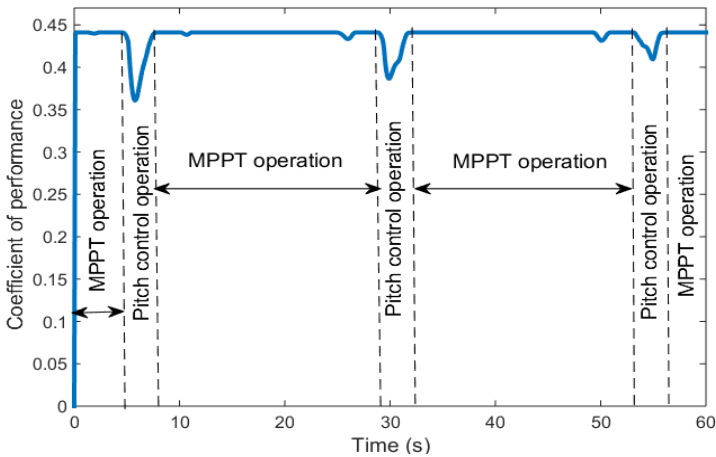

(a)

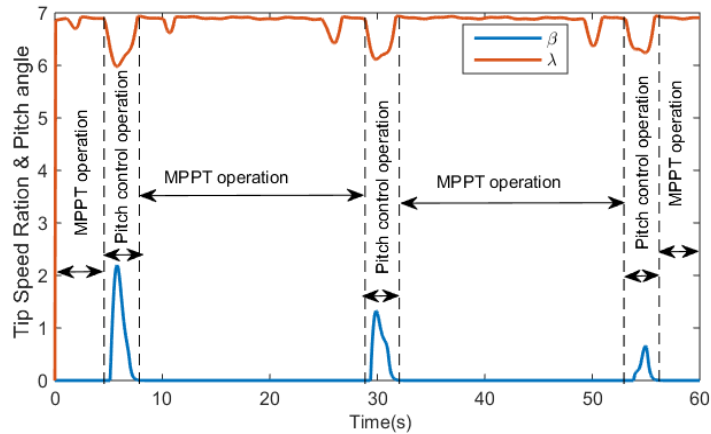

(b)

Figure 6. (a) Power coefficient, (b) Tip speed ratio and pitch angle variation

Figure 7 shows current responses in $d q$ frame, as the currents track well the reference trajectory. In this context, the quadrature current $i_{q}$ (Figure 7 (a)) varies according to the generator speed, taking into account the two wind operating modes (MPPT and pitch angle).

The direct current id (Figure 7 (b)) tracks well the optimal reference (at zero) in order to provide the maximum torque per ampere.

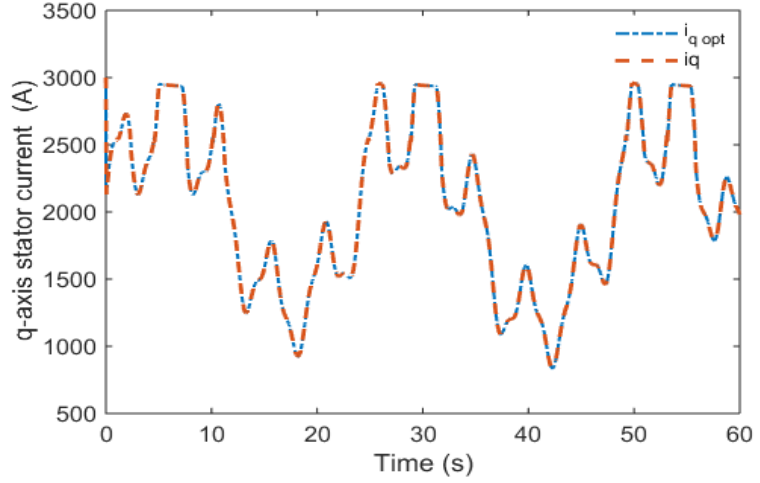

(a)

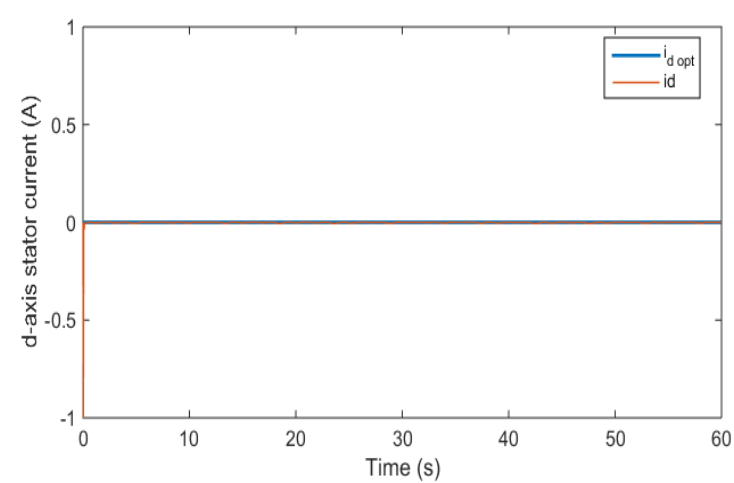

(b)

Figure 7. Stator current in d-q frame, (a) Quadrature stator current, (b) Direct stator current

Figure 8 depicts the stator currents of the PMSG in sinusoidal form. It can be noticed that stator currents have a similar shape as the wind speed during the time intervals $[0 \mathrm{~s}, 5 \mathrm{~s}]$ and also is limited, beyond the nominal wind speed as it shown during the interval [5s, $8 \mathrm{~s}]$. 
In order to guarantee the control performance of the closed-loop T-S fuzzy system, the model of the system takes into account the variation of the machine parameters $\left(R\right.$ varies by $30 \%, L_{d}=L_{q}$ varies by $20 \%$ ), that change at the same time during the time simulation.

Figure 9 shows that despite variations in the PMSG parameters, once the rated power of the generator is reached at the nominal wind speed, the robust T-S fuzzy controller track the output power at its nominal value (5 MW) accurately and rapidly even though there is uncertainty. This indicates robustness against parameter uncertainties as desired in the problem discussion. The control gains obtained are presented in Table 2.

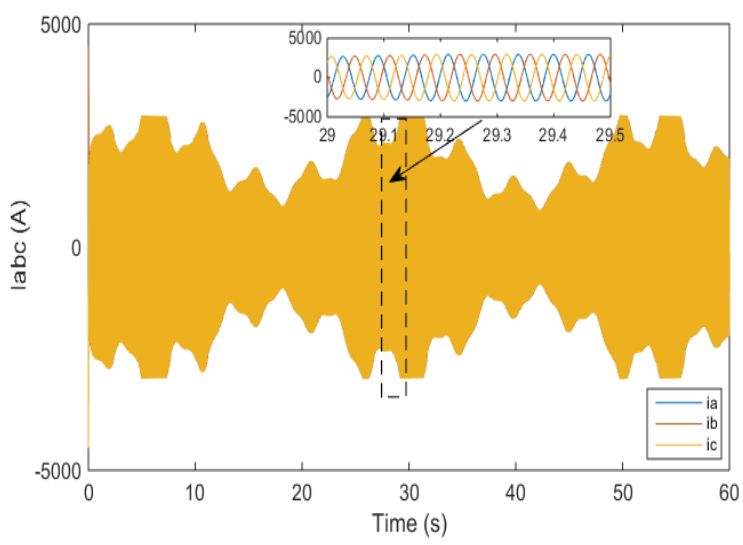

Figure 8. Stator currents Isa, Isb, Isc

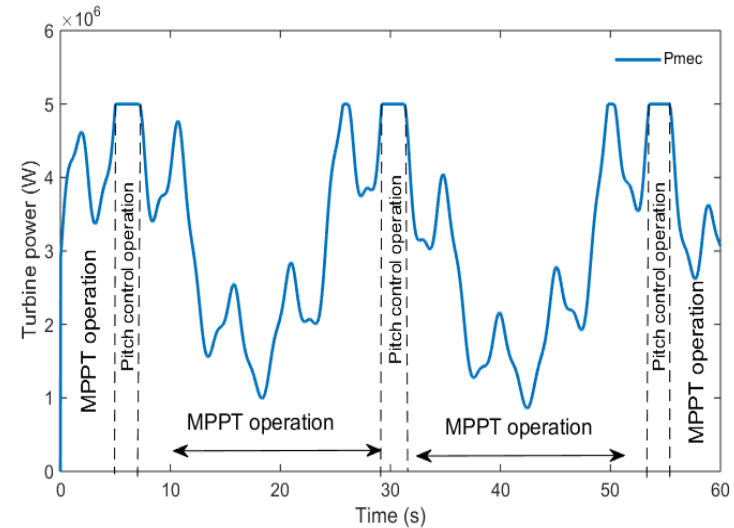

Figure 9. Turbine power response

Table 2. Feedback gains of the augmented T-S fuzzy controller

\begin{tabular}{ccc}
\hline$K_{1}=\left[\begin{array}{ccc}1080 & 0 & 2210 \\
0 & 1080 & 7480.3\end{array}\right]$ & $F_{1}=\left[\begin{array}{ccc}21.6255 & -0.1574 & -1.4084 \\
0.7070 & 53.5681 & 481.1057\end{array}\right]$ \\
$K_{2}=\left[\begin{array}{ccc}90 & 0 & 0 \\
0 & 90 & 616.3\end{array}\right]$ & $F_{2}=\left[\begin{array}{ccc}199.7734 & 0 & 0 \\
0 & 44.9273 & 398.997\end{array}\right]$ \\
\hline
\end{tabular}

\section{CONCLUSION}

This work presented a contribution to the Takagi-Sugeno fuzzy control, designed to control the variable-speed wind energy conversion system based on the PMSG. In this context, an MPPT technique was applied in order to make the system operate with maximum efficiency. Also, a pitch angle control strategy is proposed to avoid damage to the machine in strong winds so as to limit the power of the wind generator at its nominal value $(5 \mathrm{MW})$. The developed robust augmented T-S fuzzy control was designed via paralleldistributed compensation (PDC) involving system uncertainties and external disturbances. Moreover, the $H_{\infty}$ tracking control technique performance design is characterized to reject unknown disturbances. The stability of T-S fuzzy system is formulated into LMI techniques from a Lyapunov approach. The desired virtual variable is introduced to benefit the control design. The very high-performance results of numerical simulations, reveals the validity of the proposed method control in terms of robustness and saturation of the power. As a perspective, the observer-based finite time $H_{\infty}$ control will be integrated.

\section{REFERENCES}

[1] C. Zafer, et al., "Optimization of fuzzy logic (Takagi-Sugeno) blade pitch angle controller in wind turbines by genetic algorithm," Engineering Science and Technology, an International Journal IEEE transaction on Industry Applications, vol. 23, no 1, pp. 1-9, 2020.

[2] Y. Barradi et al., "Control of PMSG based variable speed wind energy conversion system connected to the grid with PI and ADRC approach," International Journal of Power Electronics and Drive Systems (IJPEDS), vol. 11, no 2, pp. 953, 2020, doi: 10.11591/ijpeds.v11.i2.pp953-968.

[3] V. Yaramasu, et al., "PMSG-based wind energy conversion systems: survey on power converters and controls," IET Electric Power Applications, vol. 11, no 6, pp. 956-968, 2017.

[4] D. Das, et al., "A $\mathrm{H}_{\infty}$ Robust Active and Reactive Power Control Scheme for a PMSG-Based Wind Energy Conversion System," IEEE Transactions on Energy Conversion, vol. 33, no 3, pp. 980-990, 2018.

[5] S. Hussien, et al., "Improving the delivered power quality from WECS to the grid based on PMSG control model," International Journal of Electrical and Computer Engineering (IJECE), vol. 10, no 6, 6349-6360, 2020, doi: 10.11591/ijece.v10i6.pp6349-6360. 
[6] R. Tiwari, et al., "Recent developments of control strategies for wind energy conversion system," Renewable and Sustainable Energy Reviews, vol 66, pp. 268-285, 2016

[7] M. Makhad, et al., "Nonlinear control of WECS based on PMSG for optimal power extraction," International Journal of Electrical and Computer Engineering (IJECE), vol. 10, no. 3, pp. 2815-2823, 2020, doi: 10.11591/ijece.v10i3.pp2815-2823.

[8] A. Dahbi, et al., "A novel combined MPPT-pitch angle control for wide range variable speed wind turbine based on neural network," International Journal of Hydrogen Energy, vol. 41, no 22, pp. 9427-9442, 2016.

[9] J. Chen, et al., "Design of robust MPPT controller for grid-connected PMSG-Based wind turbine via perturbation observation based nonlinear adaptive control," Renewable energy, vol. 134, pp. 478-495, 2019.

[10] H. Moradi, et al., "Robust control of the variable speed wind turbines in the presence of uncertainties: A comparison between Ho and PID controllers," Energy, vol. 90, pp. 1508-1521, 2015.

[11] Z. Lahlou, Zineb, et al., "Sliding mode controller based on type-2 fuzzy logic PID for a variable speed wind turbine," International Journal of System Assurance Engineering and Management, vol. 10, no 4, p. 543-551, 2019.

[12] Q-V. Ngo, et al., "The maximum power point tracking based-control system for small-scale wind turbine using fuzzy logic," International Journal of Electrical and Computer Engineering (IJECE), vol. 10, no 4, pp. 3927-3935, 2020, doi: 10.11591/ijece.v10i4.pp3927-3935.

[13] A. Iqbal, Atif, et al, "Efficacious pitch angle control of variable-speed wind turbine using fuzzy based predictive controller," Energy Reports, vol. 6, p. 423-427, 2020.

[14] Q. Ngo, et al, "The fuzzy-PID based-pitch angle controller for small-scale wind turbine," International Journal of Power Electronics and Drive Systems (IJPEDS), vol. 11, no 1, pp. 135-142, 2020, doi: 10.11591/ijpeds.v11.i1.pp135-142.

[15] M. Allouche, et al, "A Novel fuzzy Control Strategy for Maximum Power Point Tracking of Wind Energy Conversion System," International Journal of Smart Grid, vol. 3, no 3, pp. 120-127, 2019.

[16] N. Tidjani, A. Guessoum and D. Ounnas, "Permanent-Magnet Synchronous Generator Wind Turbine based on Takagi-Sugeno Fuzzy Models," 2019 7th International Renewable and Sustainable Energy Conference (IRSEC), Agadir, Morocco, 2019, pp. 1-6, doi: 10.1109/IRSEC48032.2019.9078253.

[17] M. Chinchilla, et al., "Control of permanent-magnet generators applied to variable-speed wind-energy systems connected to the grid," IEEE Transactions on energy conversion, vol. 21, no 1, pp. 130-135, 2006.

[18] J. Hussain, et al, "Adaptive maximum power point tracking control algorithm for wind energy conversion systems," EEE Transactions on Energy Conversion, vol. 31, no 2, pp. 697-705, 2016.

[19] I. Hamzaoui, et al., "Advanced control for wind energy conversion systems with flywheel storage dedicated to improving the quality of energy," International Journal of Hydrogen Energy, vol. 41, no 45, pp. 20832-20846, 2016.

[20] I. Poultangari, et al, "RBF neural network-based PI pitch controller for a class of 5-MW wind turbines using particle swarm optimization algorithm," ISA transactions, vol. 51, no 5, pp. 641-648, 2012.

[21] A. Alaboudy, et al, "Converter controls and flicker study of PMSG-based grid connected wind turbines," ISA transactions, vol. 4, no 1, pp. 1548-1556, 2005.

[22] Y. Manai, et al, "New condition of stabilisation for continuous takagi-sugeno fuzzy system based on fuzzy lyapunov function," International Journal of Control and Automation, vol. 4, no 3, pp.61-64, 2011.

[23] C. C. Kung, et al, "Tracking-based adaptive fuzzy sliding mode controller design for nonlinear systems," IET Control Theory \& Applications, vol. 1, no 1, pp.82-89, 2007.

[24] D. Ounnas, et al, "A combined methodology of $H_{\infty}$ fuzzy tracking control and virtual reference model for a PMSM," Advances in Electrical and Electronic enginnering, vol. 13, no 3, pp. 212-222, 2015.

[25] K. Y. Lian, et al, "Output tracking control for fuzzy systems via output feedback design," IEEE Transactions on Fuzzy Systems, vol. 14, no 5, pp. 628-639, 2006.

[26] M.A. Ghany, et al, "Parallel distribution compensation PID based on Takagi-Sugeno fuzzy model applied on Egyptian load frequency control," International Journal of Electrical and Computer Engineering (IJECE), vol. 10 , no 5, pp. 5274-5287, 2020, doi: 10.11591/ijece.v10i5.pp5274-5287.

[27] B. Mansouri, et al, "Output feedback LMI tracking control conditions with Hळ criterion for uncertain and disturbed T-S models," Information Sciences, vol. 179, no 4, pp. 446-457, 2009. 